Abundancia relativa y patrones de actividad de los

felinos silvestres en la selva de los Chimalapas, Oaxaca, México

\title{
Relative abundance and activity patterns of wild felids in Chimalapas rainforest, Oaxaca, Mexico
}

\author{
Miguel Briones-Salas ${ }^{1}$, Iván Lira-Torres ${ }^{2 *}$, Rogelio Carrera-Treviño ${ }^{3}$ y Gerardo Sánchez-Rojas ${ }^{4}$
}

\footnotetext{
${ }^{1}$ Laboratorio de Vertebrados Terrestres (Mastozoología), Centro Interdisciplinario de Investigación para el Desarrollo Integral Regional, Unidad Oaxaca, Instituto Politécnico Nacional. Hornos 1003, Oaxaca 71230. Oaxaca, México. Email: miguelbrionessalas@ hotmail.com (MBS).

${ }^{2}$ Instituto de Ecología Aplicada, Universidad Autónoma de Tamaulipas, Av. División del Golfo No. 356. Ciudad. Victoria 87019. Tamaulipas, México. Email: ilira_12@hotmail.com (ILT).

${ }^{3}$ Laboratorio de Fauna Silvestre, Facultad de Medicina Veterinaria y Zootecnia, Universidad Autónoma de Nuevo León, Campus Ciencias Agropecuarias, Francisco Villa s/n, Escobedo 66050. Nuevo León, México. Email: rogeliocarrera@hotmail.com (RCT).

${ }^{4}$ Instituto de Ciencias Básicas e Ingeniería, Universidad Autónoma del Estado de Hidalgo, Centro de Investigaciones Biológicas, Laboratorio de Conservación Biológica. A.P. 69, Pachuca 42001. Hidalgo, México. Email: gsanchez@uaeh.edu.mx (GSR).

*-Corresponding author.
}

The Chimalapas forests in Oaxaca, Mexico, still maintain populations of wild felids despite increased anthropogenic threats. These species have an important ecological role as predators within the carnivore assemblage. Nevertheless their distribution and population status is unknown in this region. From 2009 to 2013 we collected field data of wild felids to assess their conservation status that included presence, relative abundance, activity patterns, and population structure. We collected data during five different sampling periods using 30 camera traps per sampling period. We had a sampling effort of 9,000 trap days and estimated an index of relative abundance (IRA) of 0.44 for Herpailurus yagouaroundi, 0.55 for Leopardus wiedii, 0.66 for Puma concolor, 1.55 for Panthera onca, and 3.22 for Leopardus pardalis. L. pardalis was the species with the highest number of independent records $(\mathrm{n}=29)$ while $P$. concolor and $P$. onca were absent during the first two years of sampling. Most felid individuals were identified to gender and age class. Three types of activity patterns were recorded, with L. pardalis being active during all day ( $24 \mathrm{hrs}), H$. yagouaroundi was diurnal, while L. wiedii and $P$. onca were mostly nocturnal and $P$. concolor was crepuscular. We conclude that environmental education, regulation of subsistence hunting, establishment of a source-sink system, a livestock losses compensation program and payments for environmental services are essential for the conservation of wild felids in this priority region.

En la Selva de los Chimalapas, Oaxaca, México, aún existen poblaciones de felinos; sin embargo, debido a factores antropogénicos sus poblaciones se ven amenazadas. La importancia de estas especies se debe a la función ecológica que tienen como carnívoros depredadores, no obstante su estado poblacional y su distribución espacial en esta región son desconocidas. Durante los años 2009 al 2013, se recopilaron datos de campo sobre la presencia de estas especies en la región y sobre su estado de conservación; además, se determinaron sus abundancias relativas, patrones de actividad y estructura poblacional mediante el uso de cámaras trampa. Se Ilevaron a cabo cinco periodos de muestreo fotográficos con un total de 30 cámaras trampa en cada periodo. Con un esfuerzo total de muestreo de 9,000 días/trampa se estimó un índice de abundancia relativa (IAR) de: 0.44 para Herpailurus yagouaroundi, 0.55 para Leopardus wiedii, 0.66 para Puma concolor, 1.55 para Panthera onca y 3.22 para Leopardus pardalis. Esta última especie resultó con mayor número de registros independientes $(n=29)$, mientras $P$. concolor y $P$. onca estuvieron ausentes durante los dos primeros años de muestreo. Con respecto a la estructura poblacional se reconocieron individualmente la mayoría de los felinos manchados. Se registraron tres patrones de actividad: $H$. yagouaroundi fue una especie diurna, L. wiedii y $P$. onca fueron nocturnas, $P$. concolor una especie crepuscular, mientras que L. pardalis estuvo activa las $24 \mathrm{~h}$. Se concluye que la educación ambiental, la regulación de la cacería de subsistencia, el establecimiento de un sistema fuente-sumidero, el pago del seguro ganadero y de servicios ambientales son factores indispensables para la conservación de estas especies en la región.

Key words: Chimalapas; felines; Oaxaca; Panthera onca; Mexico.

○ 2016 Asociación Mexicana de Mastozoología, www.mastozoologiamexicana.org 


\section{Introducción}

En las últimas décadas, las actividades humanas han generado cambios muy relevantes en los ecosistemas, entre otras causas debido al establecimiento de poblados y sus consecuentes actividades, afectando las últimas regiones de importancia biológicas como es el caso de la Selva de los Chimalapas, en el estado de Oaxaca, México, considerada como uno de los sitios de mayor riqueza biológica de México y Mesoamérica (Mittermeier et al. 1999; Salas et al. 2001). Esta región incluye la mayor extensión de bosques tropicales perennifolios, subperennifolios y bosques mesófilos de montaña mejor conservados de México; además de contar con bosques caducifolios y bosques de coníferas (Lira-Torres et al. 2012). Es considerada además, una de las regiones prioritarias para la conservación del jaguar nivel I: (Ceballos et al. 2006; Briones et al. 2012). En contraste, sus habitantes enfrentan altos índices de marginación y pobreza lo cual se acentúa al tener un complejo mosaico inter étnico (zoques, tzotziles, tzeltales, chinantecos y mixtecos) al que se suman pobladores provenientes de otras regiones de Oaxaca, o bien de estados como Veracruz, Michoacán y Chiapas (Ortega del Valle et al. 2012).

La deforestación y pérdida de hábitat, conflictos agrarios, ganadería, cacería de subsistencia e incendios forestales que ocurren en los Chimalapas, amenazan la permanencia de muchas especies, entre ellas Panthera onca y otros felinos silvestres (Ortega del Valle et al. 2012; Lira et al. 2014). Los felinos desempeñan un papel ecológico primordial, pues controlan las densidades poblacionales de sus presas (Miller y Rabinowitz 2002), sin embargo a partir del año 2000 es frecuente el reporte de ganado doméstico (equinos y bovinos) depredado por felinos en los alrededores de las comunidades y en los potreros cercanos al borde de los bosques tropicales de la región, razón por la cual muchos campesinos y ganaderos eliminan a estos felinos del lugar (Lira-Torres y Ramos-Fernández 2007).

El estudio de los felinos silvestres en su hábitat natural es complicado y oneroso, incluso a niveles tan elementales como conocer su presencia en un sitio determinado (Briones et al. 2012). Su conducta sigilosa tiene como resultado que registrarlos sea poco frecuente (Gittleman et al. 2001), y si a esto se adiciona que la densidad de sus poblaciones tiende a disminuir debido a la persecución que viene derivada de la hostilidad de la gente (Rabinowitz y Nottingham 1986; Weber y Rabinowitz 1996; Sanderson et al. 2002), es necesario obtener estimaciones de su abundancia confiables, para lo cual es necesario el uso de diferentes dispositivos de captura o registro (Barea-Azcón et al. 2007).

En la selva de los Chimalapas, se han registrado previamente 149 especies de mamíferos, entre ellos 20 especies del Orden Carnívora con cinco especies de felinos: dos grandes depredadores, Puma concolor y Panthera onca, y tres mesodepredadores: Leopardus pardalis, Leopardus wiedii y Herpailurus yagouaroundi (Lira-Torres et al. 2012). Sin embargo, las poblaciones de estas especies sobre todo las especies mayores se encuentran sumamente amenazadas por la pérdida de su hábitat, la disminución de sus presas y su cacería provocada por el conflicto con la ganadería extensiva (Lavariega et al. 2013; Lira-Torres et al. 2014).

Panthera onca es una de las especies de carnívoros que más impactan al ganado en las zonas tropicales (Zimmerman et al. 2005; Zeller 2007; Rosas-Rosas et al. 2008; Peña-Mondragón 2011). Es probable que por esta razón ha desaparecido en más del 60 \% de su distribución original en México (Hoogesteijn y Hoogesteijn 2001; Ceballos et al. 2006), por lo que hoy se registra en la lista roja de especies amenazadas de la Unión Internacional para la Conservación de la Naturaleza (IUCN; Caso et al. 2008), y es considerado en México como en peligro de extinción (NOM-059ECOL-2010, SEMARNAT 2010).

Es por ello que resulta conveniente y necesario llevar a cabo estudios sobre la abundancia de las especies de felinos en estos ambientes y conocer sus tendencias poblacionales con el tiempo. 
Por lo que la presente investigación analiza la riqueza, abundancia relativa y patrones de actividad de las especies de felinos presentes en la selva de los Chimalapas, Oaxaca, México. Partiendo de la suposición de que los grandes depredadores $P$. onca y $P$. concolor requieren de mayor extensión de hábitat en buenas condiciones y son perseguidos por los pobladores locales, por lo tanto, sus abundancia relativas serán menores y sus patrones de actividad serán nocturnos; mientras que para los mesodepredadores, sus abundancias relativas serán mayores y sus patrones de actividad serán más repartidos durante las 24 horas del día.

\section{Materiales y métodos}

El trabajo se realizó dentro de los terrenos comunales de la congregación de La Fortaleza, Municipio de Santa María Chimalapa, en la región denominada como Los Chimalapas. Se ubica al sureste del

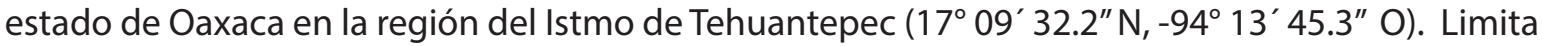
al este con la Sierra del Espinazo del Diablo, al sur con la Sierra Atravesada, ambos en Oaxaca, al norte con Los Uxpanapas, Veracruz y al oeste con el río Oaxaca (Arriaga et al. 2000).

La congregación de La Fortaleza está rodeada por montañas pedregosas y cañadas en las que predomina el bosque tropical perennifolio y subperennifolio, vegetación secundaria y pastizales introducidos (Rzedowsky 1978; Torres Colín 2004). El clima es cálido húmedo con lluvias en verano (Am(f) y (A)C(w2); García 1973). La precipitación y temperatura media anual varían de 3,000 a 3,500 $\mathrm{mm}$ y de 18 a $22^{\circ} \mathrm{C}$, respectivamente (Arriaga et al. 2000). La temporada seca abarca cuatro meses (marzo - junio) y la temporada de lluvia abarca ocho meses (julio - febrero), siendo esta región una de las más húmedas del país (Wendt 1989). Los suelos son de tipo eútrico (Alfaro 2004). El sistema hidrográfico es alimentado por las vertientes septentrionales de los ríos Oaxaca, Uxpanapa, y del escurrimiento de la zona central del río Coatzacoalcos (Ortiz Pérez et al. 2004; Figura 1).

En La Fortaleza viven cerca de 120 personas de 30 familias. Todos hablan español y algunos pobladores dominan algún idioma indígena. La mayoría de los jóvenes y adultos de la congregación se dedican a la ganadería extensiva de doble propósito, producción de carne y leche; de forma temporal cultivan maíz, frijol y calabaza. La cacería de subsistencia es una de las actividades más recurrentes en la población, pues cerca de la mitad de sus habitantes se dedican a ella (Lira-Torres y Briones-Salas 2011).

Muestreo. Se realizaron cinco temporadas de muestreo fotográfico entre los años 2009 y 2013, con una duración de 60 días cada uno. La primera se llevó a cabo durante la temporada secas del 2009 (mayo - junio). La segunda durante la temporada de lluvias 2010 (julio - agosto). La tercera durante la temporada seca (abril - mayo). La cuarta durante la temporada de lluvias (octubre noviembre) del 2012, y la quinta en la temporada de lluvias (enero - febrero) del 2013. En todas las temporadas de muestreo se colocaron 30 estaciones fijas con una cámara trampa en cada una, abarcando una superficie de 80 km² (Medellín et al. 2006; Chávez et al. 2013; Figura 1).

Las estaciones de muestreo se instalaron bajo un criterio al azar buscando siempre la cercanía a las veredas naturales, bordes de montañas, cañadas, arroyos secos y márgenes de río, separadas una de otra a una distancia de 1.5 a $3 \mathrm{~km}$ para no dejar áreas sin muestrear, y asumiendo que esta distancia es lo suficientemente grande para lograr la independencia estadística entre las estaciones de trampeo (Chávez et al. 2013). En cada estación de muestreo se instaló una cámara trampa marca Cuddeback Digital ${ }^{\oplus}$ modelo Expert, a una altura no mayor de $50 \mathrm{~cm}$ del nivel del suelo, dependiendo de la topografía e inclinación del área de muestreo. Las cámaras fueron programadas para permanecer activas durante 24 horas, mientras que el período de retardo entre fotografías fue fijado a $1 \mathrm{~min}$. La ubicación de cada una de ellas fue georreferenciada con un sistema de posicionamiento global (GPS) modelo Garmin etrex ${ }^{\circledR}$. Durante el muestreo, las cámaras trampa fueron revisadas una vez al mes; en cada fotografía detectada se imprimió la hora y fecha. 


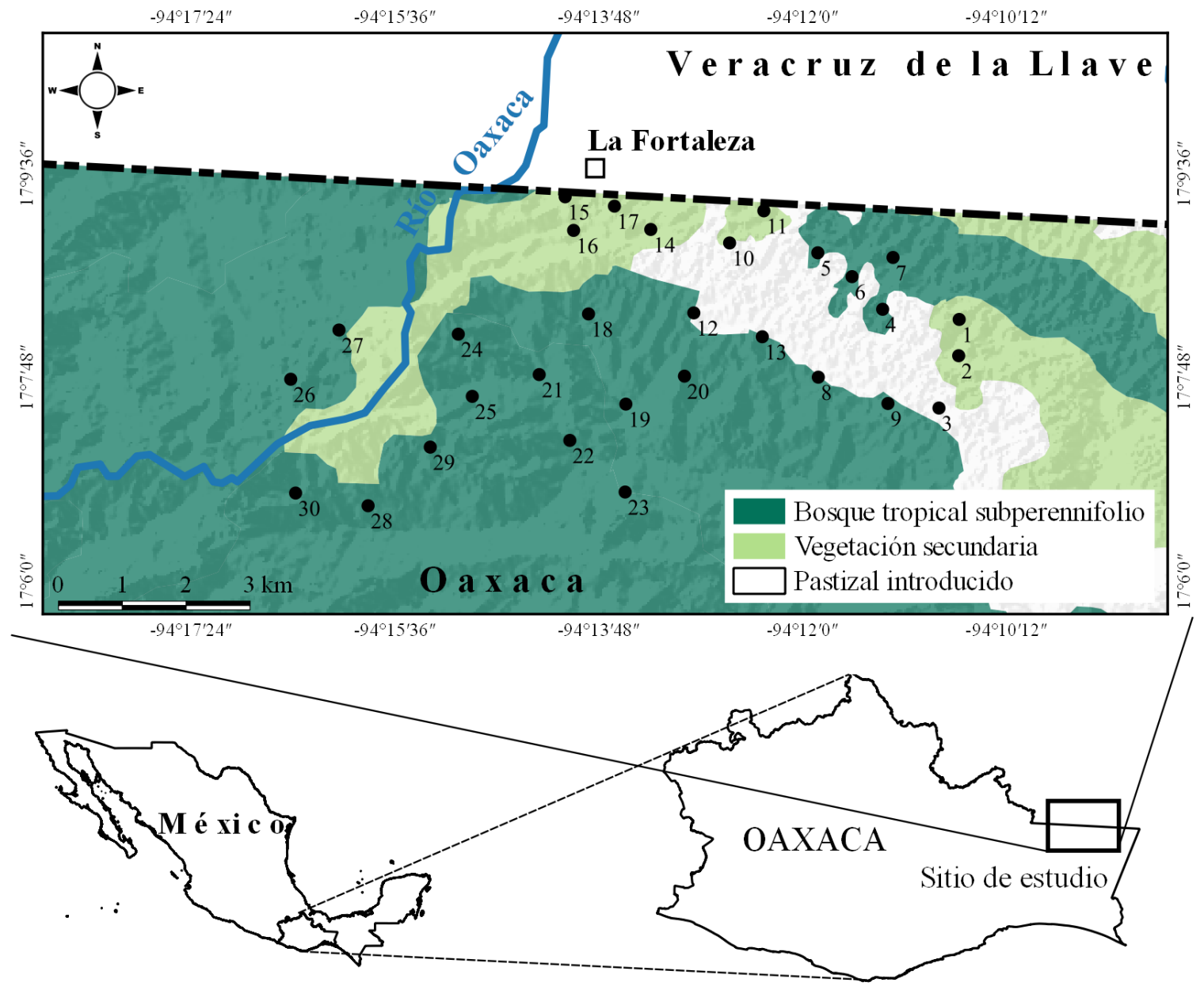

Figura 1. Sitio de estudio y distribución de las cámaras trampas en la selva de los Chimalapas, Oaxaca, México. Los tipos de vegetación presentes según los Datos Vectoriales de Uso del Suelo y Vegetación Serie IV para la Escala 1:250000. Instituto Nacional de Estadística, Geografía e Informática.

El esfuerzo total de muestreo se obtuvo multiplicando el número total de cámaras trampa por el total de días de muestreo (Medellín et al. 2006).

Abundancia relativa. Para obtener el índice de abundancia relativa (IAR) de los felinos en el área se utilizó la siguiente fórmula (Maffei et al. 2002; Sanderson 2004; Azuara 2005; Jenks et al. 2011; Zamora 2012; Lira-Torres et al. 2014): IAR $=(C / E M) * 1,000$ días/trampas.

Donde: $C$ es el número de eventos fotográficos; EM es el esfuerzo de muestreo (medido como número de cámaras multiplicado por los días de monitoreo) estacional o total y 1,000 días trampa (unidad estándar).

Se consideraron como registros fotográficos independientes los siguientes casos: a) fotografías consecutivas de diferentes individuos y/o especies y b) fotografías consecutivas de la misma especie separadas por 60 min (este criterio fue aplicado cuando no era claro si una serie de fotografías correspondían al mismo individuo, de modo que las fotografías tomadas antes de 60 min se consideraron como un solo registro; Maffei et al. 2002; Sanderson 2004; Azuara 2005; Jenks et al. 2011; Zamora 2012; Lira-Torres et al. 2014). Se comparó el IAR de cada especie de felino entre las diferentes temporadas de muestreo con una prueba de suma de rangos de Kruskal-Wallis para más de dos muestras independientes (Zar 2010).

Identificación individual. La identificación de P. onca, L. pardalis y L. wiedii se basó en el patrón de manchas en el cuerpo, que es único para cada individuo, como sucede con otros gatos manchados 
(Karanth y Nichols 1998), lo que permitió identificar diferentes individuos en la zona de estudio. Adicionalmente se trató de identificar el sexo de los individuos y si estos eran crías, jóvenes o adultos. Por otro lado, se identificó a P. concolor y $H$. yagouaroundi a partir de cicatrices que presentaban los individuos (Noss et al. 2003; Kelly et al. 2008).

Patrón de actividad. El patrón de actividad fue determinado cuando se obtuvieron al menos 10 registros fotográficos independientes de las especies (Maffei et al. 2002; Monroy-Vilchis et al. 2011; Lira-Torres et al. 2014). Los registros obtenidos se ordenaron por intervalos de una hora y se agruparon en tres periodos de tiempo: a) diurnos (09:00 -17:00 h), cuando en las fotos se observó luz solar; b) nocturnos cuando no hubo luz solar, (21:00 - 05:00 h), y c) crepusculares, cuando se obtuvieron al amanecer (05:00 - 09:00 h) o al atardecer (17:00 - 21:00 h). Se realizó una prueba de G ajustada para continuidad (Corrección de Williams) para evaluar si los registros obtenidos son independientes entre las especies de felinos y los tres periodos de tiempo (Ott 1988).

\section{Resultados}

Muestreo. El esfuerzo total de muestreo en las cinco temporadas fue de 9,000 días/trampa; 1,800 días/ trampa para cada uno de ellos, en los cuales se obtuvieron 3,058 fotografías de mamíferos grandes y medianos de la región, así como de aves. De los registros obtenidos, 58 corresponden a eventos independientes de felinos silvestres.

En este estudio se registraron cinco de las seis especies de felinos que se distribuyen en México y que se encuentran también en Norte y Sudamérica estas son: P. onca, P. concolor, L. pardalis, L. wiedii y $H$. yagouaroundi (Figura 2). De estas especies, P. onca, L. pardalis y L. wiedii se consideran en peligro de extinción de acuerdo a las normas mexicanas (NOM-059-SEMARNAT-2010, SEMARNAT 2010), además cuatro ( $P$. onca, L. pardalis, L. wiedii y H. yagouaroundi) están en la lista roja de la IUCN o en CITES. Con excepción de $L$. pardalis, la mayoría de las especies registradas solo estuvieron presentes en dos temporadas de muestreo. Los datos de registros por especie y temporada de muestreo se proporcionan en la tabla 1.

Abundancia relativa. Se encontraron diferencias significativas entre las temporadas de muestreo para las cinco especies de felinos. Para $L$. pardalis se obtuvo un IAR $=3.22$ en promedio; durante el primero y cuarto muestreo se observó el índice más alto (IAR $=6.11)$, mientras que el menor se registró durante el segundo y quinto muestreo (IAR $=0.55 ; H=13.75 ; \mathrm{g} . \mathrm{I} .=4 ; P=0.008)$. Para $L$. wiedii, se obtuvo un valor de IAR $=0.55$ en promedio; los valores resultaron mayores durante la primera y cuarta temporada de muestreo $(H=10.25 ;$ g. $I .=4 ; P=0.036)$. Para $H$. yaouaroundi fue de $I A R=0.44$ en promedio, el índice mayor se observó durante el primer muestreo, durante la segunda y quinta temporada de muestreo no se obtuvieron registros $(H=12.67 ;$ g. I. $=4 ; P=0.013$; Tabla 1$)$.

Con respecto a los felinos mayores, $P$. concolor obtuvo un IAR $=0.66$ en promedio, el mayor IAR se registró en la quinta temporada (IAR $=2.77)$. Durante tres temporadas de muestreo no se obtuvieron registros de esta especie $(H=10.759 ; \mathrm{g} . \mathrm{I} .=4 ; P=0.029)$. Para $P$. onca el IAR $=1.55$; el índice mayor se obtuvo durante el tercer muestreo (IAR $=5.55)$, mientras que durante las dos primeras temporadas no se obtuvieron registros $(H=12.404 ;$ g.l. $=4 ; P=0.155 ;$ Tabla 1$)$.

Identificación individual. Se identificaron al menos cuatro $H$. yagouaroundi adultos, uno de ellos fue macho y los otros fueron de sexo indeterminado. Cinco individuos adultos, uno macho y los otros cuatro de sexo indeterminado para $L$. wiedii. En el caso de $L$. pardalis se identificaron 22, de los cuales el $27.0 \%$ fueron machos $(n=6), 18.2 \%$ hembras $(n=4)$ y el $54.5 \%$ de sexo indeterminado $(n=12)$. Por medio del patrón de manchas se identificaron todos los ejemplares de $P$. onca, mismos que eran de sexo indeterminado. Con respecto a los ejemplares de $P$. concolor se identificaron dos individuos adultos de sexo indeterminado por medio de cicatrices y conformación anatómica, uno durante el periodo de secas del 2012 y otro durante las lluvias del 2013. 
Tabla 1. Índice de abundancia relativa (IAR) de los felinos silvestres en la selva de Los Chimalapas, Oaxaca, durante el periodo 2009-2013.

\begin{tabular}{|c|c|c|c|c|c|c|c|c|c|c|c|c|}
\hline \multirow[b]{2}{*}{ Especie } & \multirow[b]{2}{*}{$\begin{array}{l}\text { Total } \\
\text { Fotos }\end{array}$} & \multirow[b]{2}{*}{ (\%) } & \multicolumn{2}{|c|}{ Seca 2009} & \multicolumn{2}{|c|}{ Lluviosa 2010} & \multicolumn{2}{|c|}{ Seca 2012} & \multicolumn{2}{|c|}{ Lluviosa 2012} & \multicolumn{2}{|c|}{ Lluviosa 2013} \\
\hline & & & $\mathbf{N}$ & IAR & $\mathbf{N}$ & IAR & $\mathbf{N}$ & IAR & $\mathbf{N}$ & IAR & $\mathbf{N}$ & IAR \\
\hline Leopardus pardalis & 29 & 50 & 11 & 6.11 & 1 & 0.55 & 3 & 1.66 & 13 & 6.11 & 1 & 0.55 \\
\hline Leopardus wiedii & 5 & 8.62 & 2 & 1.11 & 0 & - & 1 & 0.55 & 2 & 1.11 & 0 & - \\
\hline Herpailurus yagouaroundi & 4 & 6.98 & 2 & 1.11 & 0 & - & 1 & 0.55 & 1 & 0.55 & 0 & - \\
\hline Puma concolor & 6 & 10.34 & 0 & - & 0 & - & 1 & 0.55 & 0 & - & 5 & 2.77 \\
\hline Panthera onca & 14 & 24.14 & 0 & - & 0 & - & 10 & 5.55 & 2 & 1.11 & 2 & 1.11 \\
\hline Total & 58 & 100 & & 8.33 & 1 & 0.55 & 16 & 8.86 & 18 & 8.88 & 8 & 4.43 \\
\hline Media & & & & 1.66 & & 0.11 & & 1.77 & & 1.78 & & 0.89 \\
\hline Desviación Estándar (DS) & & & & 2.54 & & 0.25 & & 2.17 & & 2.47 & & 1.15 \\
\hline Error Estándar (SE) & & & & 1.14 & & 0.11 & & 0.97 & & 1.10 & & 0.51 \\
\hline
\end{tabular}

Patrón de actividad. La prueba de G ajustada para continuidad (Corrección de Williams) para los datos de los ejemplares de $P$. concolor y $P$. onca con la finalidad de determinar la independencia entre los tres periodos de tiempo; diurnos, nocturnos y crepusculares, no fue significativa ( $\mathrm{G}=$ 6.07; $P=0.16$ ). Las tres especies de mesodepredadores (L. pardalis, L. wiedii y H. yagouaroundi) no presentan diferencias significativas $(G=8.29 ; P=0.08$ ) considerando los tres periodos de tiempo. A pesar de que no se pudieron encontrar diferencias estadísticas en el patrón de actividad de los diferentes felinos, se observan registros $(n=29)$ de $L$. pardalis como activo las $24 \mathrm{~h}$, con un pico de actividad entre 16:00 a 22:00 h. H. yagouaroundi $(n=4)$ se registró más durante el día (entre las 7:00 a las 17:00 h). L. wiedii $(n=5)$ es una especie nocturna, con actividad entre las 19:00 a 6:00 h (Fig. 3). P. concolor $(n=6)$ se registró durante el crepúsculo, con actividad entre 15:00 a 19:00 h y $P$. onca $(n=13)$ durante la noche, con una mayor actividad entre las 21:00 a 5:00 h (Figura 4).

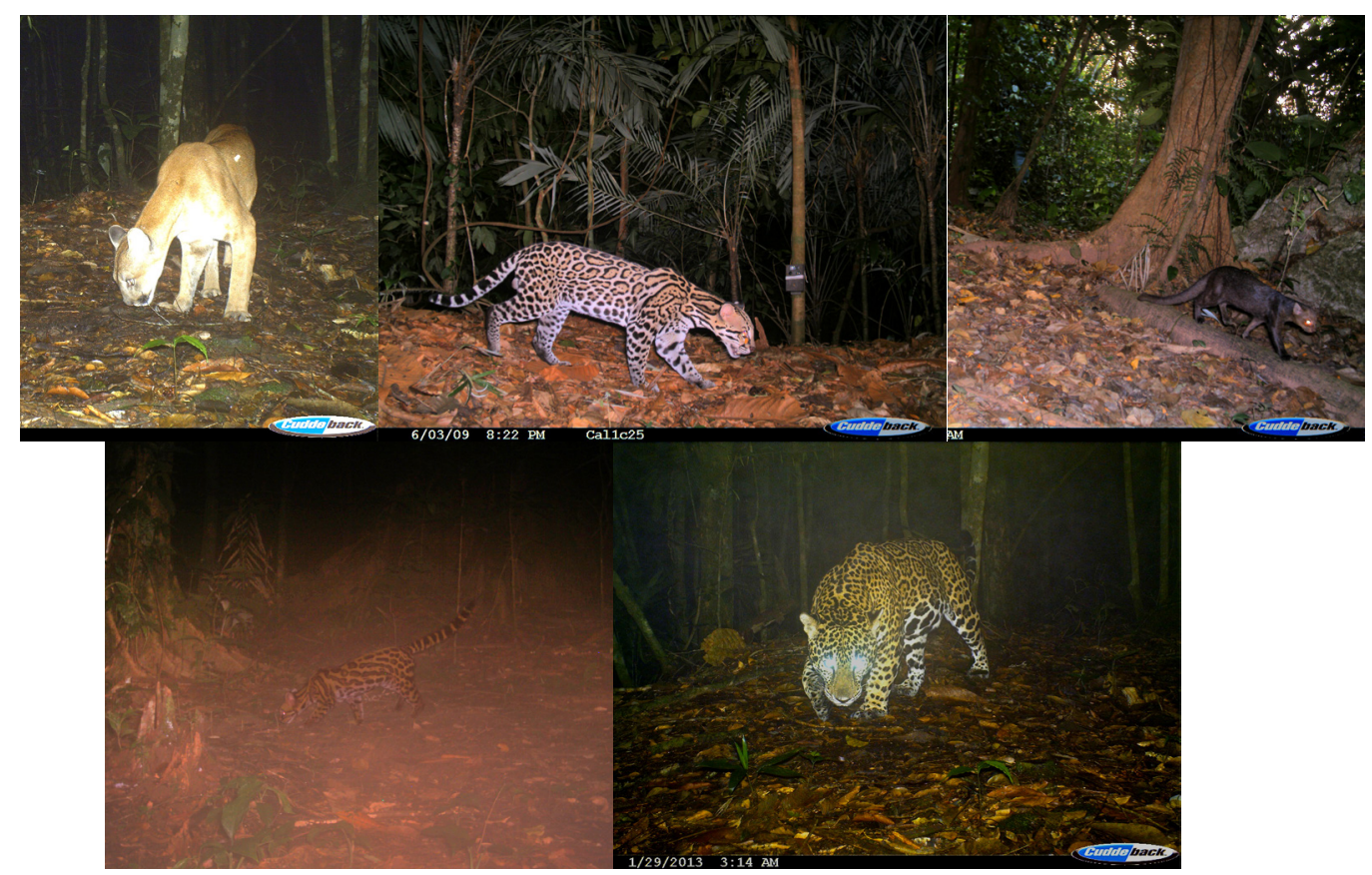

Figura 2. Imágenes de las cinco especies de felinos capturadas con foto-trampeo en los Chimalapas, Oaxaca. De izquierda a derecha: Puma concolor, Leopardus pardalis, Herpailurus yagouaroundi, Leopardus wiedii y Panthera onca. 


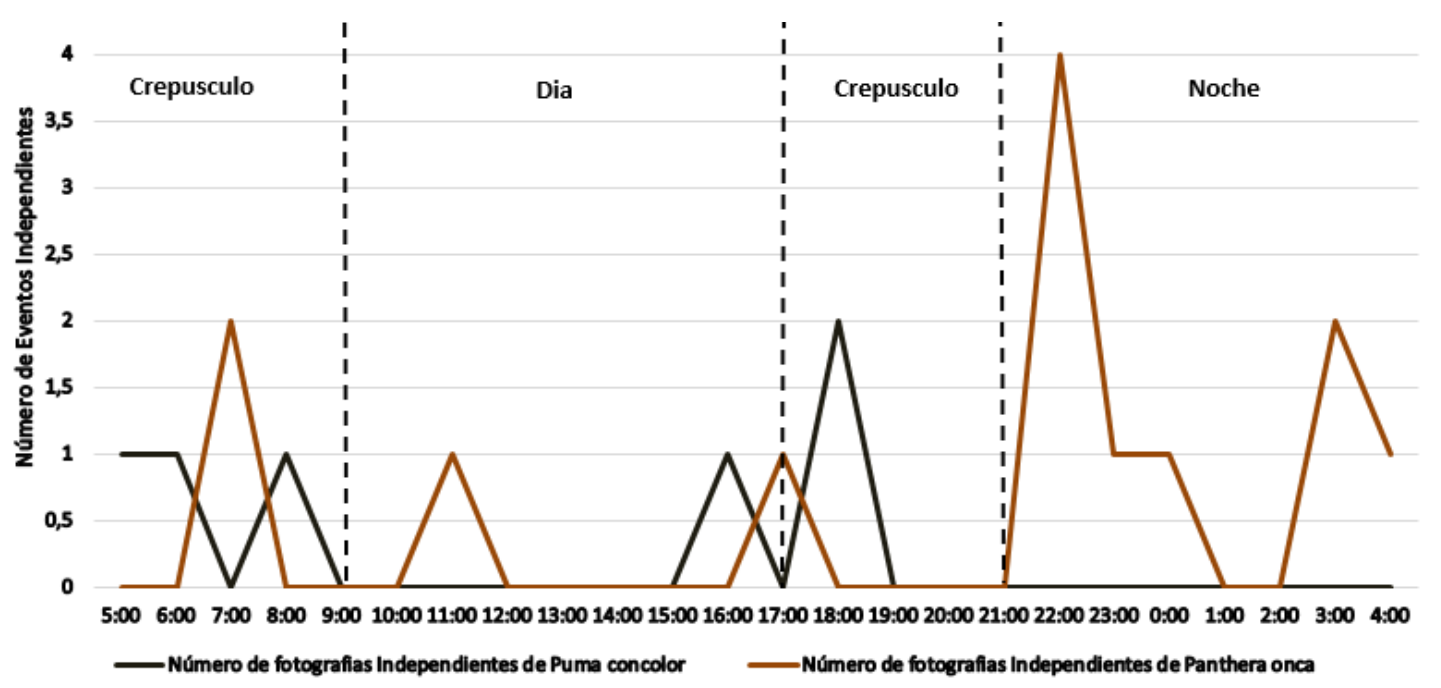

Figura 3. Patrones de actividad de P. concolor y P. onca durante los periodos de muestreo en la selva de los Chimalapas, Oaxaca, México.

\section{Discusión}

Respecto al número de fotografías independientes, el mayor tamaño de muestra se obtuvo para $L$. pardalis. La distancia entre trampas utilizadas y el tamaño del área son adecuados para estudiar a este felino (Azuara y Medellín 2007). La alta adaptabilidad a diferentes ecosistemas posiblemente sea la razón de los IAR obtenidos. Es posible que este mesodepredador se beneficie de la ausencia de grandes depredadores (Aranda 2005b), ya que los dos primeros años no fueron registrados ni $P$. concolor, ni P. onca. En el caso de L. wiedii los IAR obtenidos posiblemente estén asociados a que es una especie arborícola que rara vez baja al sustrato y por tanto nuestro método de muestreo subestime su población (Aranda 2005c). Finalmente, los bajos índices de abundancia para $H$. yagouaroundi pueden estar asociados al buen estado de conservación del área, ya que esta especie prefiere zonas de ecotonos y áreas abiertas (Aranda 2005a).

En el caso de $P$. onca y $P$. concolor durante los primeros dos años de muestreo no se obtuvieron registros fotográficos, lo que puede estar asociado a que durante esos años (2009-2010) se habían sacrificados algunos ejemplares en el área de estudio. Es posible que los registros fotográficos obtenidos a partir del tercer año, se deba a la dispersión de nuevos individuos de otras áreas del interior de la selva de los Chimalapas que llegaron a ocupar las áreas deshabitadas.

Los índices de abundancia relativa (IAR) obtenidos para L. pardalis, L. wiedii, P. concolor y $H$. yagouaroundi están por debajo de los reportados por Azuara (2005) y Azuara y Medellín (2007) y son similares para $P$. onca. Hay que tomar en cuenta que estos autores trabajaron en la estación biológica de Chajul en la zona núcleo de la Reserva de la Biosfera de Montes Azules (REBIMA) en el estado de Chiapas, un área natural protegida (ANP) donde existe poca actividad humana, todo lo contrario a nuestro sitio de estudio, donde los organismos conviven con la presencia del ser humano.

Con respecto a los patrones de actividad, $L$. pardalis es una especie que se encuentra activa durante todo el día, L. wiedii es una especie nocturna y $H$. yagouaroundi es una especie diurna. Probablemente este comportamiento está relacionado con la actividad de sus presas principales en el área (Aranda 2005a, b, ç; Vanderhoff et al. 2011; Lira-Torres y Briones-Salas 2012). Por otro lado, la diferencia en los patrones de actividad de $P$. concolor y $P$. onca posiblemente sea una estrategia para evitar encuentros entre ellos (Foster et al. 2013), ya que se han reportado varios casos de especies simpátricas con hábitos alimentarios similares donde se ha visto este tipo de comportamiento, que aparentemente disminuye la competencia y permite la coexistencia de ambos (De Almeida et al. 2004). A pesar de que no se cuenta con suficientes registros fotográficos para corroborar este aspecto, estudios indican 




Figura 4. Patrones de actividad de L. pardalis, L. wiedii y H. yagouaroundi los tres pequeños felinos silvestres durante los periodos de muestreo en la selva de los Chimalapas, Oaxaca, México.

que para evitar competencia, $P$. concolor toma una amplia variedad de tamaños de presas y más especies pequeñas que $P$. onca (Rabinowitz y Nottingham 1986; Iriarte et al. 1990; Farrell et al. 2000). Sin embargo, a pesar de que pueda existir esta separación, y que $P$. onca tiende a tomar presas grandes (Jaksic et al. 1981), en bosques en donde las presas son pequeñas y escasas $P$. onca toma lo que encuentra en proporción a su disponibilidad (Emmons 1987).

Deacuerdo a Monroy-Vilchis etal. (2007), P. concolortiene su pico de actividad duranteel crepúsculo (matutino y vespertino), lo que coincide con nuestras observaciones. Por otro lado, a partir de 121 registros obtenidos en el Parque Nacional Kaa lya en Bolivia (Maffei et al. 2007)) observaron a P. onca más activo durante el día, lo que contrasta con los datos aquí obtenidos. Sin embargo, coincide con lo reportado por Moreira et al. (2009), quien encontró que esta especie es principalmente nocturna en la Reserva de la Biosfera Maya, Petén, Guatemala. Los patrones de actividad de las principales presas potenciales: Cuniculus paca, Crax rubra, Dasypus novemcinctus, Dicotyles angulatus, Mazama temama, Nasua narica, Penelope purpurascens Tapirella bairdii, y Tayassu pecari, y reportados por LiraTorres y Briones-Salas (2012) coincide con lo observado para los dos grandes depredadores en los Chimalapas, Oaxaca, las cuales en su mayoría son de hábitos nocturnos y crepusculares.

Finalmente, los felinos silvestres son considerados especies focales por su alta sensibilidad a la cacería, a la fragmentación, pérdida de hábitat y a la disminución de sus presas potenciales. Por lo tanto, al proteger a estas especies, se garantiza la conservación de una gran cantidad de especies con las que coexiste (Miller y Rabinowitz 2002; Drury et al. 2011). Por lo que su presencia en las selvas de los Chimalapas en el estado de Oaxaca, resulta un buen indicador del estado de conservación de esta región en México.

\section{Agradecimientos}

Agradecemos el apoyo de los pobladores de la congregación La Fortaleza, Municipio de Santa María Chimalapa, Oaxaca, y del Municipio de Uxpanapa, Veracruz. De igual forma al CENJAGUAR, coordinado por el Laboratorio de Ecología y Conservación de Fauna Silvestre del Instituto de Ecología de la UNAM, al Programa Bosques Mexicanos: Selva Zoque, del Fondo Mundial para la Naturaleza (WWF por sus siglas en inglés), a la Fundación Carlos Slim y a la SIP del IPN (proyecto 
SIP: 20121142) por el financiamiento otorgado para la realización del presente estudio. M. Lavariega por la elaboración de los mapas y revisión del manuscrito. MB-S agradece a la Comisión de Operación y Fomento a las actividades Académicas (COFFA) y al programa de Estímulos al Desempeño a la Investigación (EDI), del Instituto Politécnico Nacional por el apoyo recibido. Los autores agradecen al Sistema Nacional de Investigadores (SNI) y al Programa para el Desarrollo Profesional Docente para el tipo Superior (PRODEP) por su reconocimiento y apoyo.

\section{Literatura citada}

Alfaro, S. 2004. Suelos. Pp. 55-65 en Biodiversidad de Oaxaca (García-Mendoza, A. J., M. J. Ordóñez, y M. Briones-Salas, eds.). Instituto de Biología, UNAM, Fondo Oaxaqueño para la Conservación de la Naturaleza-World Wildlife Fund. Ciudad de México, México.

Aranda, M. 2005a. Leoncillo, Jaguarundi (Herpailurus yagouroundi). Pp. 358-359 en Los Mamíferos Silvestres de México (Ceballos, G., y G. Oliva, eds.). Fondo de Cultura Económica/Comisión Nacional para el Conocimiento y Uso de la Biodiversidad. Ciudad de México, México.

Aranda, M. 2005b. Ocelote (Leopardus pardalis). Pp. 359-361 en Los Mamíferos Silvestres de México (Ceballos, G., y G. Oliva, eds.). Fondo de Cultura Económica/Comisión Nacional para el Conocimiento y Uso de la Biodiversidad. Ciudad de México, México.

Aranda, M. 2005c. Tigrillo (Leopardus wiedii). Pp. 361-362 en Los Mamíferos Silvestres de México (Ceballos, G., y G. Oliva, eds.). Fondo de Cultura Económica/Comisión Nacional para el Conocimiento y Uso de la Biodiversidad. Ciudad de México, México.

Arriaga, L., J. M. Espinoza, C. Aguilar, E. Martínez, L. Gómez, y E. Loa. 2000. Regiones terrestres prioritarias de México. Comisión Nacional para el Conocimiento y Uso de la Biodiversidad. Ciudad de México, México.

Azuara, S. D. 2005. Estimación de abundancia de mamíferos terrestres en un área de la Selva Lacandona, Chiapas. Tesis Licenciatura, Facultad de Ciencias, UNAM. Ciudad de México, México.

Azuara, D., y R. Medellin. 2007. Fototrampeo como herramienta para el estudio del jaguar y otros mamíferos en la Selva Lacandona, Chiapas. Pp. 143-153 en Conservación y Manejo del Jaguar en México: Estudios de Caso y Perspectivas (Ceballos, G., C. Chávez, R. List, y H. Zarza, eds.). CONABIO-Alianza WWF/TelcelUniversidad Nacional Autónoma de México. Ciudad de México, México.

Barea-Azcón, J. M., E. Virgós, E. Ballesteros-Duperón, M. Moleón, y M. Chirosa. 2007. Surveying carnivores at large spatial scales: a comparison of four broad-applied methods. Biodiversity and Conservation 16:1213-1230.

Briones-Salas, M., M. Lavariega, e I. Lira-Torres. 2012. Distribución actual y potencial del jaguar (Panthera onca) en Oaxaca, México. Revista Mexicana de Biodiversidad 83:246-257.

Caso, A., C. López-González, E. Payan, E. Eizirik, T. de Oliveira, R. Leite-Pitman, M. Kelly, y C. Valderrama. 2008. Panthera onca. The IUCN Red List of Threatened Species. Version 2014.2. <www.iucnredlist.org>. Downloaded on 15 September 2014.

Ceballos, G., C. Chávez, S. Blanco, R. Jiménez, M. López, O. Moctezuma, V. Támez, y M. Valdez. 2006. Áreas prioritarias para la conservación. Pp. 13-19 en El jaguar Mexicano en el siglo XXI: Situación actual y manejo (Chávez, C., y G. Ceballos, eds.). CONABIO-Alianza WWF Telcel-Universidad Nacional Autónoma de México. Ciudad de México, México.

Chávez, C., A. De la Torre, A. Bárcenas, R. Medellín, H. Zarza, y G. Ceballos. 2013. Manual de fototrampeo para estudios de fauna silvestre. El jaguar en México como estudio de caso. Alianza WWF-Telcel, Universidad Nacional Autónoma de México. Ciudad de México, México.

De Almeida, A. T., L. Silveira, y J. A. Felizola. 2004. Niche separation between the maned wolf (Chrysocyon brachyurus), the crab-eating fox (Dusicyon thus) and the hoary fox (Dusicyon vetulus) in central Brazil. Journal of Zoology 262:99-106.

Drury, R., K. Omewood, y S. Randall. 2011. Less is more: the potential of quantitative approaches in conservation research. Animal conservation 14:18-24. 
Emmons, L. 1987. Comparative feeding ecology of felids in a Neotropical rainforest. Behavioral Ecology and Sociobiology 20:271-283.

Farrell, L., J. Roman, y M. Sunquist. 2000. Dietary separation of sympatric carnivores Identified by molecular analysis of scats. Molecular Ecology 9:1583-1590.

Foster, V. C., P. Sarmento, R. Sollmann, N. Tórres, A. T. Jácomo, N. Negröes, y L. Silveira. 2013. Jaguar and puma activity patterns and predator-prey interactions in four Brazilian biomes. Biotropica 45:373379.

García, E. 1973. Modificaciones al sistema de clasificación climática de Köppen. Instituto de Geografía, Universidad Nacional Autónoma de México. Ciudad de México, México.

Gittleman, J. L., S. M. Funk, D. W. Macdonald, y R. K. Wayne. 2001. "Why Carnivore conservation"? Pp. 345-400 in Carnivore Conservation (Gittleman, J. L., S. M. Funk, D. W. Macdonald, y R. K. Wayne, eds.). Cambridge University Press. Cambridge, United Kingdom.

Hoogesteijn, R., y A. Hoogesteijn. 2001. Estrategias anti depredación para fincas ganaderas en Latinoamérica: Una guía. PANTHERA. Gráfica y Editora Microart Ltda. Campo Grande, Brasil.

IIriarte J., W. Franklin, W. Johnson, Y K. Redford. 1990. Biogeographic variation of food habits and body size of the American puma. Oecologia 85:185-190.

Jaksic, M., H. Greene, y J. Yáñez. 1981. The guild structure of a community of predatory vertebrates in central Chile. Oecologia 49:21-28.

Jenks, K. E., P. Chanteap, K. Damrongchainarong, P. Cutter, P. Cutter, T. Redford, A. J. Lynam, J. Howard, Y P. Leimgruber. 2011. Using relative abundance indices from camera-trapping to test wildlife conservation hypotheses - an example from Khao Yai National Park, Thailand. Tropical Conservation Science 4:113-131.

Karanth, K. U., Y J. D. Nichols. 1998. Estimation of tiger densities in India using Photographic captures and recaptures. Ecology 79:2852-2862.

Kelly, M. J., A. J. Noss, M. S. Di Bitetti, L. Maffei, R. L.Arispe, A. Paviolo, C. D. De Angelo, y E. Di Blanco. 2008. Estimating puma densities from camera trapping across three study sites: Bolivia, Argentina, and Belize. Journal of Mammalogy 89:408-418.

Lavariega, M. C., M. Briones-Salas, y C. Rodríguez. 2013. Registro delTapir Centroamericano (Tapirus bairdii) con cámaras-trampa en la Sierra Madre de Oaxaca, México. Revista Mexicana de Biodiversidad 84:1007-1011.

Lira-Torres, I., y G. Ramos-Fernández. 2007. El Estado del Jaguar en los Chimalapas, Oaxaca. Pp. 71-80 en Conservación y Manejo del Jaguar en México: Estudios de Caso y Perspectivas (Ceballos, G., C. Chávez, R. List, y H. Zarza, eds.). CONABIO-Alianza WWF/Telcel-Universidad Nacional Autónoma de México. Ciudad de México, México.

LiRa-Torres, I., y M. Briones-Salas. 2011. Impacto de la ganadería extensiva y cacería de subsistencia sobre la abundancia relativa de mamíferos en la Selva Zoque, Oaxaca, México. Therya 2:217-244.

Lira-Torres, I., C. Galindo-Leal, y M. Briones-Salas. 2012. Mamíferos de la Selva Zoque: riqueza, uso y conservación. Revista de Biología Tropical 60:781-797.

LiRa-Torres, I., y M. Briones-Salas. 2012. Abundancia relativa y patrones de actividad de los mamíferos de los Chimalapas, Oaxaca, México. Acta Zoológica Mexicana (n. s.) 28:566-585.

Lira-Torres, I., M. Briones-Salas, y G. SÁnchez-Rojas. 2014. Abundancia relativa, Estructura poblacional, preferencia de hábitat y patrones de actividad del Tapir Centroamericano Tapirus bairdii (Perissodactyla: Tapiridae), en la Selva de Los Chimalapas, Oaxaca, México. Revista de Biología Tropical 62:1407-1419.

Maffel, L., E. Cuellar, y J. Noss. 2002. Uso de trampas cámara para la evaluación de mamíferos en el ecotono Chaco-Chiquitanía. Revista Boliviana de Ecología y Conservación Ambiental 11:55-65.

Maffel, L., E. Cuellar, y A. Noss. 2007. Estimación de la población de jaguar con trampas cámara: Un ejemplo en Bolivia. Pp. 155-160 en Cambridge en México: Estudios de Caso y Perspectivas 
(Ceballos, G., Chávez, G., R. List, y H. Zarza, eds.). CONABIO-Alianza WWF/Telcel-Universidad Nacional Autónoma de México. Ciudad de México, México.

Medellín, R., D. Azuara, L. Maffel, H. Zarza, H. Bárcenas, E. Cruz, R. Legaria, I. Lira, G. Ramos Fernández, y S. Ávila. 2006. Censos y Monitoreo. Pp. 25-35 en El Jaguar Mexicano en el Siglo XXI: Situación Actual y Manejo (Chávez, C., y G. Ceballos, eds.). CONABIO-ALIANZA WWF TELCEL-Universidad Nacional Autónoma de México. Ciudad de México, México.

Miller, B., Y A. Rabinowitz. 2002. “¿Por qué conservar al Jaguar?” Pp. 303-315 en El Jaguar en el Nuevo Milenio (Medellín, R. A., C. Equihua, C. Chetkiewics, A. Rabinowitz, P. Crawshaw, K. Redford, J. G. Robinson, J. Sanderson, y A. Tabler, eds.). Fondo de Cultura Económica. Universidad Nacional Autónoma de México y Wildlife. Conservation Society. Ciudad de México, México.

Mittermeier, R. A., N. Myers, y C. Goettsch. 1999. Biodiversidad Amenazada; Las Ecorregiones Terrestres Prioritarias del Mundo. CEMEX, Conservación Internacional. Ciudad de México, México.

Monroy-Vilchis, O., C. Rodríguez-Soto, M. Zarco-González, y V. Urios. 2007. Distribución, uso de hábitat y patrones de actividad del puma y jaguar en el Estado de México. Pp. 59-69 en Conservación y Manejo del Jaguar en México: Estudios de Caso y Perspectivas (Ceballos, G., C. Chávez, R. List, y H. Zarza, eds.). CONABIO-Alianza WWF/Telcel-Universidad Nacional Autónoma de México. Ciudad de México, México.

Monroy-Vilchis, O., M. Zarco-González, C. Rodríguez-Soto, L. Soria-Díaz, y V. Urios. 2011. Fototrampeo de mamíferos en la Sierra Nanchititla, México. Revista de Biología Tropical 59:373-383.

Moreira, J., R. García, R. McNab, G. Ponce-Santizo, M. Mérida, y G. Ruano. 2009. Abundancia de jaguares y evaluación de presas asociadas al fototrampeo en las concesiones comunitarias del bloque de melchor de mencos, Reserva de la Biosfera Maya, Petén, Guatemala. Informe Final. Wildlife Conservation Society, Programa para Guatemala. Guatemala, Guatemala.

Noss, A., R. Cuéllas, J. Barrientos, L. Maffel, E. Cuéllar, R. Arispe, D. Rúmiz, y K. Rivero. 2003. A camara trapping and radio telemetry study of lowland tapir (Tapirus terrestris) in Bolivia dry forests. Tapir Conservation 12:24-32.

Ortega del Valle, D., L. T. Carranza, y J. Martínez. 2012. Una Mirada desde el Corazón de la Jícara de Oro (Experiencias de Conservación en la Selva Zoque de los Chimalapas). WWF - México / USAID. Oaxaca, México.

Ortiz Pérez, M. A., J. R. Hernández Santana, y J. M. Figueroa. 2004. Reconocimiento Fisiográfico y Geomorfológico. Pp. 43-54 en Biodiversidad de Oaxaca (García-Mendoza, A. J., M. J. Ordóñez, y M. Briones-Salas, eds.). Instituto de Biología, UNAM, Fondo Oaxaqueño para la Conservación de la Naturaleza-World Wildlife Fund. Ciudad de México, México.

Отт, L. 1988. An Introduction to statistical methods and data analysis, 3rd ed. PWS-Kent Publishing Company. Boston, EE.UU.

Peña-Mondragón, J. L. 2011. Daños económicos al ganado y percepciones sociales sobre el jaguar (Panthera onca veraecrucis Nelson and Goldman, 1933) en la Gran Sierra Plegada, Nuevo León, México. Tesis de maestría. Universidad Nacional Autónoma de México. Morelia, México.

Rabinowitz, A. R., y B.G. Nottingham. 1986. Ecology and behavior of the jaguar (Panthera onca) in Belize, Central America. Journal of Zoology 210:149-159.

Rosas-Rosas, O., L. C. Bender, y R. Valdez. 2008. Jaguar and puma predation on Calves in Noreastern Sonora, Mexico. Rangeland Ecology y Management 61:554-560.

Rzedowsky, J. 1978. Vegetación de México. Limusa. Ciudad de México, México.

Salas, E., C. S. Burke, C. A. Bowers, y K. A. Wilson. 2001. Team training in the skies: Does crew resource management (CRM) training work? Human Factors 43:641-674.

Sanderson E. W., C. L. Chetkiewicz, R. Medellín, A. Rabinowitz, K. H. Redford, G. Robinson, y A. B. Taber. 2002. Un análisis geográfico del estado de conservación y distribución de los jaguares a través de su área de distribución. Pp. 551-600 en El jaguar en el nuevo milenio (Medellín, R. A., C. Equihua, C. L. Chetkiewicz, P. Crawshaw, A. Rabinowitz, K. Redford, G. Robinson, E. W. Sanderson, y A. B. Taber, eds.). 
Fondo de Cultura Económica/ Universidad Nacional Autónoma de México/Wildlife Conservation Society. Ciudad de México, México.

Sanderson, J. G. 2004. Protocolo para Monitoreo con Cámaras para Trampeo Fotográfico. Tropical Ecology Assessment and Monitoring (TEAM) Initiative. The Center for Applied Biodiversity Science (CABS). Conservación Internacional. Watkinsville, EE. UU.

Secretarïa del Medio Ambiente Recursos Naturales (SEMARNAT). 2010. Norma Oficial Mexicana NOM-059ECOL-2010. Protección ambiental, especies de flora y fauna silvestres de México, categorías de riesgo y especificaciones para su inclusión, exclusión o cambio, y lista de especies en riesgo. Diario Oficial de la Federación, Jueves 30 de diciembre de 2010. 1:1-77.

Torres Colín, R. 2004. Tipos de Vegetación. Pp. 105-117 en Biodiversidad de Oaxaca (García-Mendoza, A. J., M. J. Ordoñez, y M. Briones-Salas, eds.). Instituto de Biología, UNAM, Fondo Oaxaqueño para la Conservación de la Naturaleza, World Wildlife Fund. Ciudad de México, México.

Vanderhoff, N., A. Hodge, B. Arbogast, J. Nilsson, y T. Knowles. 2011. Abundancia y patrones de actividad del margay (Leopardus wiedii) en un sitio de elevación media en los Andes Orientales de Ecuador. Mastozoología Neotropical 18:271-279.

Weber, W., y A. Rabinowitz. 1996. A global perspective on large carnivore conservation. Conservation Biology 10:1046-1054.

Wendt, T. 1989. Las Selvas de Uxpanapa, Veracruz-Oaxaca, México: Evidencia de Refugios Florísticos Cenozoicos. Anales del Instituto de Biología. Serie Botánica 58:29-54.

Zamora, J. 2012. Manual Básico de Fototrampeo: Aplicaciones al estudio de los vertebrados terrestres. Técnicas en Biología de la Conservación - № 4. Tundra Ediciones. Valencia, España.

ZaR, J. H. 2010. Biostatistical Analysis. 5th Edition. Pearson Prentice-Hall. Upper Saddle, EE. UU.

ZelLeR, K. 2007. Jaguar in the New Millenium Data Set Update: The State of Jaguar in 2006. Wildlife Conservation Society's Jaguar Conservation Program. Bronx, EE. UU.

Zimmermann, A., M. J. Walpole, y N. Leaderwilliams. 2005. Cattle ranchers' attitudes to conflicts with jaguar (Panthera onca) in the Pantanal of Brazil. Oryx 39:406-412.

Submited: August 14, 2015

Reviewed: Octaber 5, 2015

Accepted: October 20, 2015

Associated editor: Rafael Reyna 